\title{
Use of Effective Core Potential Calculations for the Conformational and Vibrational Study of Platinum(II) Anticancer Drugs. cis-Diamminedichloroplatinum(II) as a Case Study
}

\author{
Sónia M. Fiuza, Ana M. Amado,* Maria P. M. Marques, and Luis A. E. Batista de Carvalho \\ Química-Física Molecular, Departamento de Química, FCTUC, Universidade de Coimbra, P-3004-535 \\ Coimbra, Portugal \\ Received: November 14, 2007; In Final Form: January 28, 2008
}

\begin{abstract}
In the light of the recognized anticancer properties of cisplatin-type inorganic systems, the exact knowledge of their conformational preferences is of the utmost importance for understanding their biological activity. The present study reports the use of theoretical (quantum mechanical) calculations for achieving this goal. An alternative calculation method to the use of the AE basis sets, both accurate and computationally feasible, was presently tested for the conformational and vibrational study of $c i$ s-diamminedichloroplatinum(II). Effective core potentials (ECPs) were used, within the HF methodology and, within the B3LYP and mPW1PW DFT protocols. The DFT methods (particularly $\mathrm{mPW} 1 \mathrm{PW}$ ) were found to be the best choice for describing cDDP (as compared to the HF methodology).
\end{abstract}

\section{Introduction}

The discovery of cisplatin's (cis-diamminedichloroplatinum (II), cDDP) biological activity by Rosenberg ${ }^{1}$ opened the door to the use of inorganic agents as chemotherapeutic drugs. The great efficacy of cDDP led to the development and biological evaluation of a large number of related inorganic compounds, ${ }^{2,3}$ in an attempt to overcome cDDP severe side effects and/or to expand its range of activity.

At the moment, an extensive number of inorganic compounds are being subject to biological evaluation. ${ }^{4,5}$ However, prior to the biological analysis, it is important to carry out their chemical characterization mainly through spectroscopic methods, which can turn out to be a very hard task when analyzing large and complex systems. Quantum inorganic chemistry has proven to be a very helpful tool for the interpretation of the experimental spectra. $6,7,8$ However, when working with transition metal containing systems, some problems arise-not only does the number of electrons increases substantially (thus requiring a large number of two-electron integrals) but also the relativistic effects become particularly important-rendering the calculation rather costly.

In a previous publication, ${ }^{9}$ the authors have reported an extensive and systematic quantum mechanical study mainly aimed at determining the best theoretical level for describing cDDP (i.e., the best description of the structural parameters and vibrational frequencies) at the lowest computational cost. The effectiveness of Hartree-Fock (HF), density functional theory (DFT) and second-order Møller-Plesset (MP2) theoretical methods and of several standard all-electron (AE) basis sets to describe the nonmetal atoms were evaluated. On the whole, it was found that the best compromise between accuracy and computational cost is achieved at the mPW1PW/6-31G(d) level. Despite the fact that good results were obtained with this approach, the computational efforts may become prohibitive, when larger multinuclear platinum systems are considered. Hence, the requirement of an alternative calculation method that remains accurate but at the same time allows the applicability to more complex systems is fundamental.

* Corresponding author: Telephone/Fax: +351-239 826541. E-mail: ama1@portugalmail.pt.
In the present work, the Effective Core Potentials $(\mathrm{ECPs})^{10}$ approximation is applied in order to perform a conformational and vibrational study of cDDP, as an alternative to the use of the AE basis sets. The study includes both HF and B3LYP and mPW1PW DFT methodologies. The MP2 theoretical method is not considered, since it implies a large computational effort and was previously shown to have no significant advantages over the other methods. ${ }^{9}$

\section{Experimental Methods}

2.1. Computational Details. All the calculations were performed on a personal computer, using the Gaussian $03 \mathrm{~W}$ (G03W) package. ${ }^{11}$ In view of the allowed free rotation of the amine groups, three geometries - two $C_{2 v}$ (cDDP1 and cDDP3) and one $C_{s}(\mathrm{cDDP} 2)$-were considered (Figure 1). All attempts in order to optimize geometries with all the hydrogen bonds lying out of the $\mathrm{Pt}-\mathrm{Cl}$ plane failed, as they converged to one of those three conformers.

All geometries were fully optimized by the Berny algorithm, using redundant internal coordinates, within symmetry constrains. The optimization convergence criteria for the cutoffs of forces and step sizes considered were as follows: 0.000015 Hartree/Bohr for maximum force, 0.000010 Hartree/Bohr for root-mean-square force, $0.000060 \mathrm{Bohr}$ for maximum displacement, and $0.000040 \mathrm{Bohr}$ for root-mean-square displacement $(\mathrm{G} 03 \mathrm{~W}$ keyword $o p t=t i g h t)$. In all cases, vibrational frequency calculations were performed, at the same theory level, in order to confirm that the geometries correspond to a real minimum at the potential energy surface (no negative eigenvalues) as well as to quantify the zero-point vibrational energy correction (zpve).

Description of the nitrogen and chloride atoms was based on two standard non-splitted valence ECPs (as implemented in G03W program package) by using the keywords $L A N L 2 M B$ (which considers the all-electron STO-3G ${ }^{12}$ basis set for the nitrogen and the ECP of Hay and Wadt ${ }^{13}$ for the chloride) and $C E P-4 G$ (which uses the ECPs of Stevens et al. ${ }^{14}$ for both nitrogen and chloride atoms). The effect of splitting both the nitrogen and the chloride valence shells was considered by using either CEP-31 $\mathrm{G}^{14}$ or LANL2DZ keywords (this option uses the D95 $\mathrm{V}^{15}$ basis set for nitrogen and the ECPs of Hay and Wadt ${ }^{13}$ 

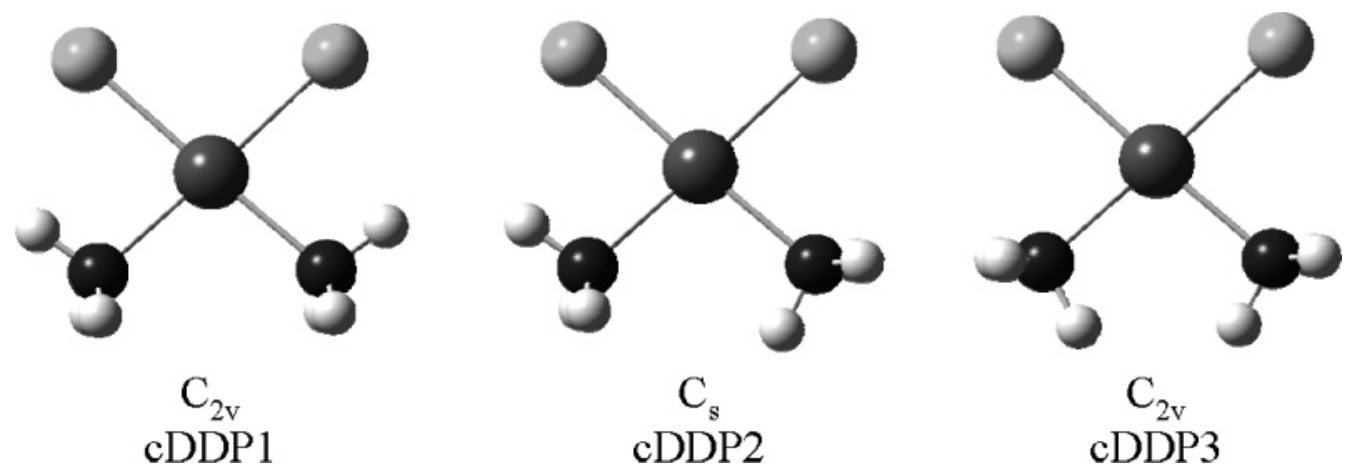

Figure 1. Schematic representation of the optimized geometries for cDDP.

TABLE 1: List of the Theoretical Levels Considered in This Study for the Pt Ligands, as Well as the Corresponding Nomenclature Used Throughout the Text and Tables

\begin{tabular}{llll}
\hline theoretical level $^{a}$ & nomenclature & \multicolumn{1}{c}{ ECP } & nomenclature \\
\hline HF & HF & LANL2MB & ECP1 \\
B3LYP & B3L & LANL2DZ & ECP2 \\
mPW1PW & mPW1 & CEP-4G & ECP3 \\
& & CEP-31G & ECP4
\end{tabular}

${ }^{a}$ In all cases LANL2DZ ${ }^{16} \mathrm{ECP}$ was considered for the Pt atom and the standard AE basis set 3-21G for the hydrogen atoms. ${ }^{b}$ For basis set details refer to ref $12\left(\mathrm{~N}\right.$ atom) and ref $13\left(\mathrm{Cl}\right.$ atom). ${ }^{c}$ For basis set details refer to ref $15\left(\mathrm{~N}\right.$ atom) and ref $13\left(\mathrm{Cl}\right.$ atom). ${ }^{d}$ For basis set details refer to ref 14 ( $\mathrm{N}$ and $\mathrm{Cl}$ atoms).

in a double- $\zeta$ splitting for chloride). For the description of the $\mathrm{H}$ and $\mathrm{Pt}$ atoms, the theoretical levels previously reported ${ }^{9}$ were applied-the standard AE 3-21G basis set and the relativistic pseudopotential of Hay and Wadt ${ }^{16}$ (G03W keyword LANL2DZ), respectively.

The effect of considering a polarization function at the Ptatom was also tested, by augmenting the valence shell with an f-function $\left(\xi_{\mathrm{Pt}}=0.993\right) .{ }^{17}$ Moreover, introduction of polarization functions on both nitrogen and chloride atoms was taken into account, by including a d-polarization function in the corresponding valence shells $\left(\xi_{\mathrm{N}}=0.8\right.$ and $\left.\xi_{\mathrm{Cl}}=0.75\right) .{ }^{18}$

In order to evaluate the effect of electron correlation (known to be particularly important in systems containing transition metal atoms ${ }^{19}$ ), different theory methods were considered. Thus, all ECP combinations were tested within HF and two different DFT protocols: (i) the widely used B3LYP, which includes a mixture of HF and DFT exchange terms and the gradientcorrected functionals of Lee, Yang and Parr, as proposed and parametrized by Becke; ${ }^{20,21}$ (ii) mPW1PW, which considers a modified version of the exchange term of Perdew-Wang and the Perdew-Wang 91 correlation functional. ${ }^{22,23}$ For each of these DFT approaches, the effect of two different integration grids was assessed: (i) a pruned grid of 75 radial shells and 302 angular points per shell (G03W keyword grid=75302, specifying a FineGrid), and (ii) a pruned grid of 99 radial shells and 590 angular points per shell (G03W keyword grid $=99590$, defining an UltraFineGrid).

The different theoretical approaches and ECPs combinations are collected in Table 1, along with the corresponding nomenclature used throughout the text and Tables.

2.2. Theoretical Methods and Evaluation of the Accuracy Level. The aim of the present work is to develop a theoretical approach coupling the best possible theoretical description of the experimental data available for cDDP, to a low computational cost. Similar to the former AE study on cDDP, ${ }^{9}$ a comparison between the calculated values of both structural parameters and vibrational frequencies and the corresponding
TABLE 2: Calculated Relative Energies $\left(\mathrm{kJ} \mathrm{mol}^{-1}\right)$ for the Three Conformations of cDDP (Figure 1), Using the Different ECPs, Augmented with a d-Polarization Function at the Nitrogen and Chloride Atoms, and LANL2DZ, Augmented with an f-Polarization Function at the Valence Shell of the Pt Atom

\begin{tabular}{llll}
\hline theoretical level $^{a}$ & \multicolumn{1}{c}{$\mathrm{cDDP}^{b}$} & $\mathrm{cDDP}^{b}$ & $\mathrm{cDDP}^{b}$ \\
\hline HF-ECP1 & 0.00 & 0.72 & 1.83 \\
HF-ECP2 & 0.00 & 0.81 & 2.19 \\
HF-ECP3 & $0.00(0.00)$ & $0.08(0.20)$ & 0.83 \\
HF-ECP4 & $0.02(0.00)$ & $0.00(0.28)$ & $0.46(0.53)$ \\
B3L-ECP1 & 0.00 & 4.08 & 8.34 \\
B3L-ECP2 & 0.00 & 3.85 & 8.13 \\
B3L-ECP3 & 0.00 & 2.71 & 5.98 \\
B3L-ECP4 & 0.00 & 2.64 & 5.64 \\
mPW1-ECP1 & 0.00 & 3.95 & 8.18 \\
mPW1-ECP2 & 0.00 & 3.52 & 7.58 \\
mPW1-ECP3 & 0.00 & 2.32 & 5.30 \\
mPW1-ECP4 & 0.00 & 2.31 & 5.09
\end{tabular}

${ }^{a}$ In the case of the DFT results, the values are those obtained by using the FineGrid option. ${ }^{b}$ The calculated values after zpve energy correction are presented in parenthesis when the conformation was found to be a real minimum at the potential energy surface.

experimental values was performed (for further details on this accuracy evaluation procedure, please refer to ref 9).

\section{Results and Discussion}

3.1. Conformational Analysis. Table 2 presents the bare relative energies calculated for the three cDDP optimized geometries using the different ECPs augmented polarization functions at all the heavy atoms. Tables S2a, S2b, S2c, and S2d of the Supporting Information comprise, respectively, the energetic results obtained with ECPs plus d-polarization functions at the $\mathrm{N}$ and $\mathrm{Cl}$ atoms, f-polarization function at the $\mathrm{Pt}$ center, without polarization functions, and using the UltraFineGrid option without polarization functions in the case of the DFT calculations. As previously found in the AE calculations, ${ }^{9}$ an improvement of the Integration Grid does not affect the $\Delta E$ values between conformations (Table S2c vs Table S2d).

Table 2 shows that the DFT protocols are unanimous in predicting a single minimum-cDDP1-for the cDDP isolated molecule, as previously found. ${ }^{9}$ Removal of either the fpolarization function at the Pt-atom (Table S2a) or the dpolarization functions at the $\mathrm{N}$ - and $\mathrm{Cl}$-valence shells (Table S2b) were not found to affect the final result. On the other hand, concerning the magnitude of the calculated energy differences between conformations, it is evident that inclusion of an f-polarization function at the metal has a negligible effect (Table 2 vs Table S2a, or Table S2b vs S2c). A decrease of the $\Delta E$ values is generally observed upon introduction of d-polarization functions in the $\mathrm{Cl}-$ and $\mathrm{N}$-valence shells (Table S2b vs Table 2, or Table S2c vs Table S2a). The only exception is observed with ECP4, as considering a d-polarization function to describe 
TABLE 3: Calculated Structural Parameters (Distances in pm and Angles in deg) for the Lowest-Energy Minimum Predicted for cDDP (geometry cDDP1), Using the Different ECPs, Augmented with a d-Polarization Function at the Nitrogen and Chloride Atoms, and LANL2DZ, Augmented with an f-Polarization Function at the Valence Shell, of the Pt Atom

\begin{tabular}{|c|c|c|c|c|c|c|c|c|c|c|c|}
\hline \multirow{2}{*}{$\frac{\text { theoretical level }^{a}}{\text { HF-ECP1 }}$} & \multicolumn{2}{|c|}{$\mathrm{Pt}-\mathrm{Cl}^{b}$} & \multicolumn{2}{|c|}{$\mathrm{Pt}-\mathrm{N}^{b}$} & \multicolumn{2}{|c|}{$\mathrm{Cl}-\mathrm{Pt}-\mathrm{Cl}^{b}$} & \multicolumn{2}{|c|}{$\mathrm{Cl}-\mathrm{Pt}-\mathrm{N}^{b}$} & \multicolumn{2}{|c|}{$\mathrm{N}-\mathrm{Pt}-\mathrm{N}^{b}$} & \multirow{2}{*}{$\frac{\Delta \Delta^{c}}{5.5}$} \\
\hline & 236.1 & 3.1 & 209.5 & 9.5 & 96.2 & 4.3 & 84.2 & -6.1 & 95.4 & 8.4 & \\
\hline HF-ECP2 & 235.4 & 2.4 & 211.6 & 11.6 & 96.2 & 4.3 & 84.2 & -6.1 & 95.4 & 8.4 & 5.6 \\
\hline HF-ECP3 & 235.4 & 2.4 & 212.3 & 12.3 & 95.4 & 3.5 & 85.0 & -5.3 & 94.5 & 7.5 & 5.1 \\
\hline HF-ECP4 & 235.4 & 2.4 & 211.9 & 11.9 & 95.5 & 3.6 & 84.8 & -5.5 & 94.9 & 7.9 & 5.2 \\
\hline B3L-ECP1 & 234.7 & 1.7 & 208.9 & 8.9 & 95.7 & 3.8 & 82.8 & -7.5 & 98.7 & 11.7 & 6.2 \\
\hline B3L-ECP2 & 234.1 & 1.1 & 210.2 & 10.2 & 95.7 & 3.8 & 82.9 & -7.3 & 98.6 & 11.6 & 6.3 \\
\hline B3L-ECP3 & 234.4 & 1.4 & 211.4 & 11.4 & 94.5 & 2.6 & 84.1 & -6.2 & 97.3 & 10.3 & 5.6 \\
\hline B3L-ECP4 & 234.3 & 1.2 & 210.6 & 10.6 & 95.0 & 3.1 & 83.6 & -6.7 & 97.8 & 10.8 & 5.8 \\
\hline mPW1-ECP1 & 232.3 & -0.8 & 206.6 & 6.6 & 95.4 & 3.5 & 83.1 & -7.2 & 98.5 & 11.5 & 5.8 \\
\hline mPW1-ECP2 & 231.7 & -1.4 & 207.6 & 7.6 & 95.3 & 3.4 & 83.2 & -7.1 & 98.4 & 11.4 & 5.8 \\
\hline mPW1-ECP3 & 232.0 & -1.1 & 209.0 & 9.0 & 94.3 & 2.4 & 84.3 & -6.0 & 97.1 & 10.1 & 5.2 \\
\hline mPW1-ECP4 & 231.8 & -1.2 & 208.0 & 8.0 & 94.7 & 2.8 & 83.9 & -6.3 & 97.6 & 10.6 & 5.4 \\
\hline
\end{tabular}

${ }^{a}$ In the case of the DFT results, the values are those obtained by using the FineGrid option. ${ }^{b}$ Difference between calculated and experimental values in boldface; in the case of $\mathrm{Pt}-\mathrm{Cl}, \mathrm{Pt}-\mathrm{N}$ and $\mathrm{Cl}-\mathrm{Pt}-\mathrm{N}$, the average of the two experimental values was used. The experimental individual values are as follows: $\mathrm{Pt}-\mathrm{Cl}, 233.3$ and $232.8 \mathrm{pm} ; \mathrm{Pt}-\mathrm{N}, 195.0$ and $205.0 \mathrm{pm} ; \mathrm{Cl}-\mathrm{Pt}-\mathrm{N}, 88.5^{\circ}$ and $92.0^{\circ} ; \mathrm{Cl}-\mathrm{Pt}-\mathrm{Cl}, 91.9^{\circ} ; \mathrm{N}-\mathrm{Pt}-\mathrm{N}, 87.0^{\circ} .{ }^{26}$ ${ }^{c}$ Overall mean percent difference obtained by the equation: ${ }^{9} \Delta \Delta=\left[\left(\sum_{i=1}^{n}\left|\Delta_{i}\right| / x_{i}\right) / n\right] \times 100$, where $\Delta_{i}=($ theoretical value - experimental value $)$ is the individual deviation and $\mathrm{x}_{\mathrm{i}}$ the experimental value.

the $\mathrm{N}$ and $\mathrm{Cl}$ atoms leads to an increase of the $\Delta E$ values within both DFT protocols (Table S2b vs Table 2 or Table S2c vs Table S2a). However, this result seems to be in better conformity with the ones obtained with the other DFT/ECP combinations.

When considering the change of ECP within each theoretical level, it can be observed that the splitting of the $\mathrm{N}$ - and $\mathrm{Cl}$ valence shells $(\mathrm{ECP} 1 \rightarrow \mathrm{ECP} 2$ and $\mathrm{ECP} 3 \rightarrow \mathrm{ECP} 4)$ leads to a general decrease of the $\Delta E$ values. Both the f-polarization function in the Pt-valence shell and the type of integration grid have a minor effect on the magnitude of the $\Delta E$ values.

As for the AE basis sets, ${ }^{9}$ the presently reported $\mathrm{HF}$ results are strongly dependent on the type of ECP considered. For the ECPs of Hay and Wadt ${ }^{13}$ (HF-ECP1 and HF-ECP2), a single minimum (cDDP1) is predicted, and the splitting of the valence shell (HF-ECP1 $\rightarrow$ HF-ECP2) causes an increase of the conformational energy differences. ECP improvement through inclusion of polarization functions does not change the stability order between conformers, affecting only the magnitude of the $\Delta E$ values. In turn, when the ECPs of Stevens et al. ${ }^{14}$ (HFECP3 and HF-ECP4) are used, more than one conformation is predicted to be a real minimum. If no polarization function is considered (Table S2c) ECP3 and ECP4 yield distinct global minima. In other words, the splitting of the valence shell (ECP3 $\rightarrow$ ECP4) leads to a change of the lowest energy conformer from cDDP1 to cDDP3. Regarding this cDDP1/cDDP3 relative stability, addition of an f-polarization function to the metal valence shell is found to be irrelevant (Table S2b). On the other hand, inclusion of polarization functions at both the $\mathrm{N}$ - and $\mathrm{Cl}$ valence shells is mandatory, as it renders cDDP1 the global minimum at both HF-ECP3 and HF-ECP4 basis sets (Tables S2a and 2).

On the whole, these results show that the use of effective core potentials for the study of cDDP-like systems requires the inclusion of polarization functions at the nonmetal heavy atoms, mainly when using HF approaches. In contrast, the effect of considering f-polarization functions at the platinum atom is negligible. In the case of the DFT calculations, improvement of the integration grid is also negligible.

3.2. Structural Analysis. As for the AE accuracy evaluation, ${ }^{9}$ the following structural and vibrational analysis is solely performed for the cDDP1 geometry, since this is either the only real minima or the lowest energy conformer yielded by the different theoretical approaches used.

Table 3 lists the optimized structural parameters calculated by the four ECP methodologies augmented with d-polarization functions at the $\mathrm{N}$ - and $\mathrm{Cl}$ atoms, and LANL2DZ plus an f-polarization function at the Pt-valence shell. The $\Delta$-values for each structural parameter, as well as the overall $\Delta \Delta$-values determined for each ECP combination, are represented in boldface (for definition of $\Delta$ - and $\Delta \Delta$-values see ${ }^{9}$ and the footnote of Table 3). All other results, obtained using dpolarization functions at both the $\mathrm{N}$ - and $\mathrm{Cl}$ atoms, augmented with an f-polarization function to describe the Pt center, no polarization functions, or the UltraFineGrid option, are comprised in the Supporting Information (Tables S3a, S3b, S3c, and $\mathrm{S} 3 \mathrm{~d}$, respectively).

As expected, the lowest overall mean percent deviations $(\Delta \Delta$ values) are obtained when using polarization functions at all heavy atoms. The overall accuracy increases according to the sequence: no polarization function $($ Table S3c) $\rightarrow$ polarization function at the metal atom (Table S3b) $\rightarrow$ polarization functions at the $\mathrm{N}$ and $\mathrm{Cl}$ atoms (Table S3a) $\rightarrow$ polarization functions at all heavy atoms (Table 3). The highest accuracy improvement is obtained by inclusion of d-polarization at the $\mathrm{N}$ - and Cl-valence shells (Table S3b $\rightarrow$ Table 3 or Table S3c $\rightarrow$ Table $\mathrm{S} 3 \mathrm{a}$ ), which leads to improvements in the range of $0.3-1.1 \%$, while addition of an f-polarization function at the metal valence shell (Table S3a $\rightarrow$ Table 3 or Table S3c $\rightarrow$ Table S3b) is responsible for improvements not higher than $0.3 \%$. Considering both types of polarization functions (Table S3c $\rightarrow$ Table 3) yields an overall improvement approximately equivalent to the sum of the individual improvements.

It is interesting to note that when d-polarization functions are considered for describing the $\mathrm{N}$ and $\mathrm{Cl}$ atoms, the valence shell splitting is avoidable (ECP1 $\rightarrow$ ECP2 and ECP3 $\rightarrow$ ECP4). In fact, the valence shell splitting leads to variations of the $\Delta \Delta$ values not larger than $0.5 \%$ (Table $\mathrm{S} 3 \mathrm{c}$ ), while addition of polarization functions to describe these atoms (Table S3b $\rightarrow$ Table 3 or Table S3c $\rightarrow$ Table S3a) yields an accuracy enhancement higher than $0.8 \%$ for all ECPs but one (ECP4 for which the improvements are $0.2-0.5 \%$ ). Hence, from an overall accuracy point of view, ECP3 is the best choice when dpolarization functions are added at the $\mathrm{N}$ and $\mathrm{Cl}$ atoms. Otherwise, the corresponding splitted valence shell (ECP4) should be chosen. Finally, the best overall accuracy is achieved through the use of the mPW1PW protocol, independently of the complexity of the $\mathrm{N}$ - and $\mathrm{Cl}$-valence shells. On the whole, the mPW1/ECP3(d)/LanL2DZ theoretical level seems to be the best choice.

At this stage, the efficiency of the different theoretical levels to describe each structural parameter individually should be evaluated, knowing in advance that any improvement of the 
integration grid in the DFT calculations has no influence in this predicting ability.

Bond Distances. Regarding the $\mathrm{Pt}-\mathrm{Cl}$ distance, it is evident that the description of this structural parameter generally improves in the sequence observed for the overall mean percent deviation ( $\Delta \Delta$-values): no polarization function (Table S3c) $\rightarrow$ polarization function the metal atom (Table S3b) $\rightarrow$ polarization functions at the $\mathrm{N}$ and $\mathrm{Cl}$ atoms (Table S3a) $\rightarrow$ polarization functions at all heavy atoms (Table 3 ). The only exception is observed for the mPW1PW results, for which a clear worsening is usually observed when going from polarization functions at the $\mathrm{N}$ and $\mathrm{Cl}$ atoms to polarization functions at all heavy atoms (Table S3a vs Table 3). While the reasons for this behavior are still not clear, it should be noticed that the effect of the addition of an f-polarization function at the metal is quite small when compared to the effects of the addition of d-polarization functions at the $\mathrm{N}$ and $\mathrm{Cl}$ atoms, independently of the ECP and the theoretical method considered (either HF or DFT). In other words, the largest improvement in the description of the $\mathrm{Pt}-\mathrm{Cl}$ parameter is achieved by inclusion of polarization functions to describe the $\mathrm{N}$ and $\mathrm{Cl}$ atoms (Table $\mathrm{S} 3 \mathrm{~b} \rightarrow$ Table 3 or Table S3c $\rightarrow$ Table S3a).

As to the $\mathrm{Pt}-\mathrm{N}$ distance, the three methods used lead to overestimated distances, regardless of the ECP improvements introduced and of the theoretical protocol used. In contrast to the $\mathrm{Pt}-\mathrm{Cl}$ bond, the use of $\mathrm{f}$-polarization functions to describe the platinum valence shell corresponds to an ECP improvement that leads to the best theoretical-to-experimental agreement (Table S3a $\rightarrow$ Table 3 or Table S3c $\rightarrow$ Table S3b). Inclusion of a d-polarization function at the $\mathrm{N}$ and $\mathrm{Cl}$ atoms leads to a general worsening of the $\mathrm{Pt}-\mathrm{N}$ description (Table $\mathrm{S} 3 \mathrm{~b} \rightarrow$ Table 3 or Table S3c $\rightarrow$ Table S3a), this effect being more pronounced for ECP1.

Concerning the choice of the ECP, it can be verified that the ECPs of Hay and Wadt, ${ }^{20}$ in a minimal valence splitting scheme (ECP1) yield the best accuracy, in all cases. Furthermore, as verified for the $\mathrm{Pt}-\mathrm{Cl}$ distance, the mPW1PW DFT protocol yields the lowest deviations between theory and experiment for all ECPs tested, independently of the valence shell improvements adopted.

Bond Angles. In agreement with the results obtained by the $\mathrm{AE}$ basis sets, ${ }^{9}$ all ECPs presently used yield overestimated $\mathrm{Cl}-$ $\mathrm{Pt}-\mathrm{Cl}$ and $\mathrm{N}-\mathrm{Pt}-\mathrm{N}$ angles, and underestimated $\mathrm{Cl}-\mathrm{Pt}-\mathrm{N}$ values, within all three considered theoretical protocols. This may result from the formation of intramolecular $\mathrm{N}-\mathrm{H} \cdots \mathrm{Cl}$ close contacts, which lead to a decrease of the $\mathrm{Cl}-\mathrm{Pt}-\mathrm{N}$ angle and concomitantly to an opening of the other bond angles within the molecule. In order to account for the effects of these interactions, calculations concerning the dimeric structures held by $\mathrm{N}-\mathrm{H} \cdots \mathrm{Cl}$ intermolecular contacts, and evidenced in the $\mathrm{X}$-ray structure reported in the literature, are currently understudy. This will hopefully allow a better representation of the structural parameters of cDDP (and of other small Pt(II) complexes), in particular of the bond angles.

The largest deviations detected are, once again, observed for the $\mathrm{N}-\mathrm{Pt}-\mathrm{N}$ angles, while the best theoretical-to-experimental agreement occurs for the $\mathrm{Cl}-\mathrm{Pt}-\mathrm{Cl}$ values. Moreover, the best prediction of the latter is obtained with the mPW1PW protocol that yields slightly better results than B3LYP. On the contrary, the other two bond angles are better described within the HF methodology. These discrepancies are probably related to the different weight given by the two theoretical protocols (HF and DFT) to the hydrogen bonding interactions occurring in the system, either attractive $(\mathrm{N}-\mathrm{H} \cdots \mathrm{Cl})$ or repulsive $(\mathrm{H} \cdots \mathrm{H}$ and
TABLE 4: Computational Time (CPUT) Required for a Vibrational Frequency Calculation Using the Different ECPs at the Nonmetal Atoms and the LANL2DZ ECP at the Pt $\operatorname{Atom}^{a}$

\begin{tabular}{lcccc}
\hline & \multicolumn{4}{c}{ CPUT $^{b}$} \\
\cline { 2 - 5 } theoretical level & no/no & yes/no & no/yes & yes/yes \\
\hline HF-ECP1 & $00: 20$ & $00: 38$ & $00: 34$ & $02: 10$ \\
HF-ECP2 & $00: 27$ & $02: 33$ & $01: 41$ & $03: 11$ \\
HF-ECP3 & $00: 20$ & $00: 38$ & $00: 36$ & $02: 12$ \\
HF-ECP4 & $00: 24$ & $02: 18$ & $00: 44$ & $02: 57$ \\
B3L-ECP1 & $06: 28$ & $10: 26$ & $08: 14$ & $12: 44$ \\
B3L-ECP2 & $09: 07$ & $14: 37$ & $11: 33$ & $16: 32$ \\
B3L-ECP3 & $06: 24$ & $09: 40$ & $08: 24$ & $12: 53$ \\
B3L-ECP4 & $07: 46$ & $14: 25$ & $10: 01$ & $17: 13$ \\
mPW1-ECP1 & $06: 22$ & $10: 06$ & $08: 19$ & $12: 54$ \\
mPW1-ECP2 & $09: 16$ & $14: 02$ & $11: 44$ & $16: 48$ \\
mPW1-ECP3 & $06: 29$ & $09: 41$ & $08: 30$ & $13: 01$ \\
mPW1-ECP4 & $07: 48$ & $14: 27$ & $10: 05$ & $17: 13$
\end{tabular}

${ }^{a}$ no/no (no polarization functions), yes/no (d-polarization functions at $\mathrm{N}-$ and $\mathrm{Cl}$ atoms), no/yes (f-polarization function at the $\mathrm{Pt}$ atom), yes/yes (polarization functions at all heavy atoms). ${ }^{b}$ Computational time required for a frequency calculation, min:sec.

$\mathrm{Cl} \cdots \mathrm{Cl}$ ). This weighting clearly defines which bond angles will open and which will close during the optimization process, relative to the perfect square planar geometry (all bond angles equal to $90{ }^{\circ} \mathrm{C}$ ). Using ECP3 as an example (since it yields the lowest rms value Table 3 ), the corresponding $\mathrm{N}-\mathrm{H} \cdots \mathrm{Cl}$ distances are $257.5 \mathrm{pm}$ (HF-ECP3), $250.3 \mathrm{pm}$ (B3L-ECP3), and $248.5 \mathrm{pm}$ (mPW1-ECP3), clearly showing that these intermolecular close contacts become more important for the DFT protocols, particularly for mPW1PW.

The results obtained along this work also indicate that meaningful improvements in the description of the cDDP bond angles are only achieved by adding polarization functions at the $\mathrm{N}$ - and $\mathrm{Cl}$-valence shells while the inclusion of an $\mathrm{f}$ polarization function at the metal has an almost negligible effect (Table S3c $\rightarrow$ Table S3a vs Table S3c $\rightarrow$ Table S3b and Table $\mathrm{S} 3 \mathrm{a} \rightarrow$ Table 3). Addition of d-polarization functions to the valence shells of the nonmetal heavy atoms, in turn, renders the minimal ECP3 more accurate than the corresponding double- $\zeta$ splitted scheme (ECP4). On the whole, the predicting accuracy for the three cisplatin bond angles increases in the order: $\mathrm{ECP} 4 \rightarrow \mathrm{ECP} 4+f$-polarization function (at the metal) $\rightarrow \mathrm{ECP} 3+d$-polarization functions (at the $N$ and $\mathrm{Cl}$ atoms). It is thus possible to conclude that the best overall agreement for cDDP (lowest $\Delta \Delta$-value) is achieved with the mPW1PW functional coupled to either the ECP3 or ECP4 approaches (with or without d-polarization at the nonmetal heavy atoms, respectively). Addition of f-polarization functions to the ECP describing the platinum center (LANL2DZ) leads only to slight improvements, which are more pronounced for the description of the $\mathrm{Pt}-\mathrm{N}$ distance.

In view of attaining a final decision on the best choice for a suitable theoretical methodology for representing this type of cisplatin-like molecules, an evaluation of their efficacy for predicting the corresponding vibrational frequencies of cDDP must be undertaken.

3.3. Vibrational Analysis. Table 4 lists the computational time required for a vibrational frequency calculation for cDDP, at the different theoretical levels tested. As expected, both the inclusion of polarization functions and splitting of the $\mathrm{N}$ - and Cl-valence shells are responsible for an increase of the computational time. This CPUT (central processor unit time) increase is larger when d-polarization functions are added to describe both $\mathrm{N}$ and $\mathrm{Cl}$ atoms than when considering an f-polarization function at the metal. For all the ECPs tested, 
TABLE 5: Best Grouping of the cDDP Vibrational Modes, Set Scaling Factors $\left(\lambda_{\mathrm{g}}\right)$ and Rms Values Obtained Using the ECPs Considered at the Nonmetal Atoms and LANL2DZ at the Pt Atom

\begin{tabular}{|c|c|c|c|c|c|c|c|c|c|}
\hline \multirow[b]{2}{*}{ theoretical level $^{c}$} & \multirow[b]{2}{*}{ funct. $^{d}$} & \multicolumn{3}{|c|}{ modes within the set ${ }^{a}$} & \multicolumn{3}{|c|}{$\lambda \mathrm{g}^{b}$} & \multirow[b]{2}{*}{$\mathrm{rms}^{e}$} & \multirow[b]{2}{*}{$\mathrm{cpu}^{f}$} \\
\hline & & 1 & 2 & 3 & 1 & 2 & 3 & & \\
\hline HF-ECP1 & no/no & $\begin{array}{l}v \mathrm{NH}_{3} \\
\delta \mathrm{NH}_{3}\end{array}$ & $\begin{array}{l}\rho \mathrm{NH}_{3} \\
\delta \mathrm{N}-\mathrm{Pt}-\mathrm{Cl}\end{array}$ & $\begin{array}{l}v \mathrm{Pt}-\mathrm{N} \\
v \mathrm{Pt}-\mathrm{Cl} \\
\delta \mathrm{N}-\mathrm{Pt}-\mathrm{N} \\
\delta \mathrm{Cl}-\mathrm{Pt}-\mathrm{Cl}\end{array}$ & 0.85 & 0.93 & 1.04 & $1.1 \%$ & 00:20 \\
\hline HF-ECP2 & no/no & $\begin{array}{l}v \mathrm{NH}_{3} \\
\delta \mathrm{NH}_{3}\end{array}$ & $\begin{array}{l}\rho \mathrm{NH}_{3} \\
\nu \mathrm{Pt}-\mathrm{Cl} \\
\delta \mathrm{N}-\mathrm{Pt}-\mathrm{Cl}\end{array}$ & $\begin{array}{l}v \mathrm{Pt}-\mathrm{N} \\
\delta \mathrm{N}-\mathrm{Pt}-\mathrm{N} \\
\delta \mathrm{Cl}-\mathrm{Pt}-\mathrm{Cl}\end{array}$ & 0.88 & 0.96 & 1.10 & $1.8 \%$ & $00: 27$ \\
\hline HF-ECP3 & no/yes & $\begin{array}{l}\nu \mathrm{NH}_{3} \\
\delta \mathrm{NH}_{3}\end{array}$ & $\begin{array}{l}\rho \mathrm{NH}_{3} \\
\delta \mathrm{N}-\mathrm{Pt}-\mathrm{Cl}\end{array}$ & $\begin{array}{l}\nu \mathrm{Pt}-\mathrm{N} \\
\nu \mathrm{Pt}-\mathrm{Cl} \\
\delta \mathrm{N}-\mathrm{Pt}-\mathrm{N} \\
\delta \mathrm{Cl}-\mathrm{Pt}-\mathrm{Cl}\end{array}$ & 0.87 & 0.94 & 1.07 & $1.6 \%$ & $00: 36$ \\
\hline HF-ECP4 & no/no & $\begin{array}{l}v \mathrm{NH}_{3} \\
\delta \mathrm{NH}_{3}\end{array}$ & $\begin{array}{l}\rho \mathrm{NH}_{3} \\
\nu \mathrm{Pt}-\mathrm{Cl} \\
\delta \mathrm{N}-\mathrm{Pt}-\mathrm{Cl}\end{array}$ & $\begin{array}{l}\nu \mathrm{Pt}-\mathrm{N} \\
\delta \mathrm{N}-\mathrm{Pt}-\mathrm{N} \\
\delta \mathrm{Cl}-\mathrm{Pt}-\mathrm{Cl}\end{array}$ & 0.87 & 0.95 & 1.08 & $1.9 \%$ & 00:24 \\
\hline B3L-ECP1 & no/no & $\begin{array}{l}v \mathrm{NH}_{3} \\
\delta_{\text {as }} \mathrm{NH}_{3} \\
\delta \mathrm{N}-\mathrm{Pt}-\mathrm{Cl}\end{array}$ & $\begin{array}{l}\delta_{\mathrm{s}} \mathrm{NH}_{3} \\
\rho \mathrm{NH}_{3}\end{array}$ & $\begin{array}{l}\nu \mathrm{Pt}-\mathrm{N} \\
\nu \mathrm{Pt}-\mathrm{Cl} \\
\delta \mathrm{N}-\mathrm{Pt}-\mathrm{N} \\
\delta \mathrm{Cl}-\mathrm{Pt}-\mathrm{Cl}\end{array}$ & 0.93 & 0.98 & 1.05 & $1.3 \%$ & $06: 28$ \\
\hline B3L-ECP2 & no/no & $\begin{array}{l}v \mathrm{NH}_{3} \\
\delta_{\text {as }} \mathrm{NH}_{3} \\
\delta \mathrm{N}-\mathrm{Pt}-\mathrm{Cl}\end{array}$ & $\begin{array}{l}\delta_{\mathrm{s}} \mathrm{NH}_{3} \\
\rho \mathrm{NH}_{3} \\
\nu \mathrm{Pt}-\mathrm{Cl}\end{array}$ & $\begin{array}{l}\nu \mathrm{Pt}-\mathrm{N} \\
\delta \mathrm{N}-\mathrm{Pt}-\mathrm{N} \\
\delta \mathrm{Cl}-\mathrm{Pt}-\mathrm{Cl}\end{array}$ & 0.94 & 1.00 & 1.10 & $1.7 \%$ & 09:07 \\
\hline B3L-ECP3 & no/yes & $\begin{array}{l}\nu \mathrm{NH}_{3} \\
\delta_{\text {as }} \mathrm{NH}_{3} \\
\delta \mathrm{N}-\mathrm{Pt}-\mathrm{Cl}\end{array}$ & $\begin{array}{l}\delta_{\mathrm{s}} \mathrm{NH}_{3} \\
\rho \mathrm{NH}_{3} \\
\nu \mathrm{Pt}-\mathrm{Cl} \\
\alpha \mathrm{Cl}-\mathrm{Pt}-\mathrm{Cl}\end{array}$ & $\begin{array}{l}\nu \mathrm{Pt}-\mathrm{N} \\
v \mathrm{Pt}-\mathrm{Cl}\end{array}$ & 0.95 & 0.99 & 1.08 & $1.8 \%$ & $08: 24$ \\
\hline B3L-ECP4 & no/no & $\begin{array}{l}\nu \mathrm{NH}_{3} \\
\delta_{\text {as }} \mathrm{NH}_{3} \\
\delta \mathrm{N}-\mathrm{Pt}-\mathrm{Cl}\end{array}$ & $\begin{array}{l}\delta_{\mathrm{s}} \mathrm{NH}_{3} \\
\rho \mathrm{NH}_{3} \\
\nu \mathrm{Pt}-\mathrm{Cl}\end{array}$ & $\begin{array}{l}\nu \mathrm{Pt}-\mathrm{N} \\
\delta \mathrm{N}-\mathrm{Pt}-\mathrm{N} \\
\delta \mathrm{Cl}-\mathrm{Pt}-\mathrm{Cl}\end{array}$ & 0.94 & 1.00 & 1.10 & $1.4 \%$ & $07: 46$ \\
\hline mPW1-ECP1 & no/no & $\begin{array}{l}\nu \mathrm{NH}_{3} \\
\delta_{\text {as }} \mathrm{NH}_{3} \\
\delta \mathrm{N}-\mathrm{Pt}-\mathrm{Cl}\end{array}$ & $\begin{array}{l}\delta_{\mathrm{s}} \mathrm{NH}_{3} \\
\rho \mathrm{NH}_{3}\end{array}$ & $\begin{array}{l}\nu \mathrm{Pt}-\mathrm{N} \\
\nu \mathrm{Pt}-\mathrm{Cl} \\
\delta \mathrm{N}-\mathrm{Pt}-\mathrm{N} \\
\delta \mathrm{Cl}-\mathrm{Pt}-\mathrm{Cl}\end{array}$ & 0.91 & 0.96 & 1.01 & $1.5 \%$ & $06: 22$ \\
\hline mPW1-ECP2 & no/no & $\begin{array}{l}\nu \mathrm{NH}_{3} \\
\delta_{\text {as }} \mathrm{NH}_{3} \\
\nu \mathrm{Pt}-\mathrm{Cl} \\
\delta \mathrm{N}-\mathrm{Pt}-\mathrm{Cl}\end{array}$ & $\begin{array}{l}\delta_{\mathrm{s}} \mathrm{NH}_{3} \\
\rho \mathrm{NH}_{3}\end{array}$ & $\begin{array}{l}\nu \mathrm{Pt}-\mathrm{N} \\
\delta \mathrm{N}-\mathrm{Pt}-\mathrm{N} \\
\delta \mathrm{Cl}-\mathrm{Pt}-\mathrm{Cl}\end{array}$ & 0.93 & 0.98 & 1.05 & $1.8 \%$ & 09:16 \\
\hline mPW1-ECP3 & no/yes & $\begin{array}{l}\nu \mathrm{NH}_{3} \\
\delta_{\text {as }} \mathrm{NH}_{3} \\
\delta \mathrm{N}-\mathrm{Pt}-\mathrm{Cl}\end{array}$ & $\begin{array}{l}\delta_{s} \mathrm{NH}_{3} \\
\rho \mathrm{NH}_{3} \\
\nu \mathrm{Pt}-\mathrm{Cl} \\
\delta \mathrm{Cl}-\mathrm{Pt}-\mathrm{Cl}\end{array}$ & $\begin{array}{l}\nu \mathrm{Pt}-\mathrm{N} \\
\delta \mathrm{N}-\mathrm{Pt}-\mathrm{N}\end{array}$ & 0.93 & 0.99 & 1.07 & $1.6 \%$ & $08: 30$ \\
\hline mPW1-ECP4 & no/no & $\begin{array}{l}\nu \mathrm{NH}_{3} \\
\delta_{\text {as }} \mathrm{NH}_{3} \\
\nu \mathrm{Pt}-\mathrm{Cl} \\
\delta \mathrm{N}-\mathrm{Pt}-\mathrm{Cl}\end{array}$ & $\begin{array}{l}\delta \mathrm{sNH}_{3} \\
\rho \mathrm{NH}_{3}\end{array}$ & $\begin{array}{l}\nu \mathrm{Pt}-\mathrm{N} \\
\delta \mathrm{N}-\mathrm{Pt}-\mathrm{N} \\
\delta \mathrm{Cl}-\mathrm{Pt}-\mathrm{Cl}\end{array}$ & 0.94 & 0.98 & 1.06 & $1.5 \%$ & $07: 48$ \\
\hline
\end{tabular}

${ }^{a} \nu_{\mathrm{s}}$ and $\nu_{\mathrm{as}}$ stand for symmetric and antisymmetric stretching modes, respectively; $\delta_{\mathrm{s}}$ and $\delta_{\mathrm{as}}$ stand for symmetric and antisymmetric deformation modes, respectively; $\delta=$ skeletal in-plane deformation modes; $\rho$ and $\rho^{\prime}$ stand for in-phase and out-of-phase rocking modes, respectively. For a schematic representation of the $\delta \mathrm{N}-\mathrm{Pt}-\mathrm{N}, \delta \mathrm{Cl}-\mathrm{Pt}-\mathrm{N}$, and $\delta \mathrm{Cl}-\mathrm{Pt}-\mathrm{Cl}$ modes refer to Figure 3 of reference $9 .{ }^{b}$ Calculated with equation ${ }^{9} \lambda_{s}=$ $\left(\sum_{i=1}^{n_{g}} \lambda_{i}\right) / n_{s .}{ }^{c}$ In the case of the DFT results, the values are those obtained by using the FineGrid option. ${ }^{d}$ no/no (no polarization functions), yes/no (d-polarization functions at $\mathrm{N}$ and $\mathrm{Cl}$ atoms), no/yes (f-polarization function at the $\mathrm{Pt}$ atom), yes/yes (polarization fuctions at all heavy atoms)-using the FineGrid option in the DFT calculations. ${ }^{e}$ Calculated with the following equation: ${ }^{9} \mathrm{rms}=\left(\sum_{i=1}^{n} \Delta_{i}\right) / n .{ }^{f}$ Computational time required for a frequency calculation, min:sec.

the DFT calculations were found to be more time-consuming than the HF ones.

In general, the vibrational frequencies $(\omega)$ are overestimated relative to the experimental ones $(v)$, which justifies the common practice of scaling the theoretical values. While extensive work has been carried out for organic compounds in order to standardize these scaling factors, ${ }^{24}$ no studies are to be found on this subject for inorganic systems. In the authors' previous work, ${ }^{9}$ different scaling approaches were tested and assessed for cisplatin, leading to distinct scaling factors for each allelectrons theoretical level considered. A similar evaluation is presented in this study, for the different ECP combination schemes tested.

The use of a single scaling factor (Tables S5a, S5b, S5c, and S5d) is not suitable, since it yields relatively high rms values (indicative of a low accuracy), independently of the theory level used. An increase in the number of scaling factors to two (one for the vibrational modes above $400 \mathrm{~cm}^{-1}$ and another for those predicted below $400 \mathrm{~cm}^{-1}$ ) leads to a modest accuracy improvement (Tables S5e, S5f, S5g, and S5h). In order to obtain a significant decrease of the rms values, a three-factor approach was found to be required. The results yielded by this approach, for the different theoretical levels considered, are compiled in Tables S5i, S5j, S5k, and S51. The grouping scheme of the different vibrational modes was subject to a trial and error process in view of achieving the best approach, corresponding to the smallest overall error (lowest rms-see ref 9 for more details).

Grouping of the vibrational modes within the three frequency sets is dependent on the ECP approximation, on the valence shell improvement considered (with or without polarization functions) and on the theoretical method used. Addition of polarization functions to the $\mathrm{N}$ - and $\mathrm{Cl}$-valence shells generally leads to an increase of the overall error (increase of the rms values) in all cases but four (Table S51 vs Table S5j). Exceptions are observed when using ECP2 and ECP3, for both DFT protocols.

Table 5 presents the best grouping scheme determined for the cDDP vibrational modes, the corresponding scaling factors, and the CPUT for each theoretical level considered. Based on 
TABLE 6: Calculated Vibrational Frequencies of cDDP and Comparison with the Experimental Ones, after Scaling in Accordance with Table 5

\begin{tabular}{|c|c|c|c|c|c|c|c|}
\hline \multirow{3}{*}{$\begin{array}{l}\text { vibrational } \\
\text { mode }^{a}\end{array}$} & \multicolumn{7}{|c|}{ theoretical level } \\
\hline & \multicolumn{2}{|c|}{ HF-ECP1 } & \multicolumn{2}{|c|}{ B3L-ECP1 } & \multicolumn{3}{|c|}{ mPW1-ECP1 } \\
\hline & $\omega^{b}$ & $\Delta^{c}$ & $\omega^{d}$ & $\Delta^{c}$ & $\omega^{e}$ & $\Delta^{c}$ & $v^{f}$ \\
\hline$v_{\mathrm{as}} \mathrm{NH}_{3}$ & 3358 & 49 & 3344 & 35 & 3360 & 51 & 3309 \\
\hline$\nu_{\mathrm{as}} \mathrm{NH}_{3}$ & 3345 & 59 & 3310 & 24 & 3320 & 34 & 3287 \\
\hline$v_{\mathrm{s}} \mathrm{NH}_{3}$ & 3193 & -18 & 3152 & -59 & 3162 & -49 & 3211 \\
\hline$\delta_{\mathrm{as}} \mathrm{NH}_{3}$ & 1622 & -27 & 1623 & -26 & 1611 & -38 & 1648 \\
\hline$\delta^{\prime}{ }_{\mathrm{a}} \mathrm{NH}_{3}$ & 1614 & -14 & 1616 & -12 & 1604 & -24 & $1628^{g}$ \\
\hline$\delta_{\mathrm{as}} \mathrm{NH}_{3}$ & 1593 & -8 & 1590 & -11 & 1579 & -22 & 1601 \\
\hline$\delta^{\prime}{ }_{\text {as }}^{\prime} \mathrm{NH}_{3}$ & 1586 & 49 & 1672 & 47 & 1573 & 36 & 1537 \\
\hline$\delta_{\mathrm{s}} \mathrm{NH}_{3}$ & 1290 & -26 & 1317 & 1 & 1309 & -7 & 1316 \\
\hline$\delta_{\mathrm{s}}^{\prime} \mathrm{NH}_{3}$ & 1284 & -11 & 1313 & 18 & 1305 & 10 & 1295 \\
\hline$\rho \mathrm{NH}_{3}$ & 870 & 32 & 861 & 23 & 862 & 24 & 824 \\
\hline$\rho^{\prime} \mathrm{NH}_{3}$ & 821 & -3 & 829 & 5 & 829 & 5 & 811 \\
\hline$\rho \mathrm{NH}_{3}$ & 803 & -7 & 790 & -20 & 794 & -16 & 789 \\
\hline$\rho^{\prime} \mathrm{NH}_{3}$ & 775 & -14 & 773 & -16 & 776 & -13 & 724 \\
\hline$v_{\mathrm{s}} \mathrm{Pt}-\mathrm{N}$ & 521 & -3 & 522 & -2 & 525 & 1 & 524 \\
\hline$v_{\mathrm{as}} \mathrm{Pt}-\mathrm{N}$ & 508 & 0 & 514 & 6 & 516 & 8 & 508 \\
\hline$v_{\mathrm{s}} \mathrm{Pt}-\mathrm{Cl}$ & 327 & 4 & 328 & 5 & 329 & 6 & 323 \\
\hline$v_{\text {as }} \mathrm{Pt}-\mathrm{Cl}$ & 315 & -1 & 316 & 0 & 317 & 1 & 317 \\
\hline$\delta \mathrm{N}-\mathrm{Pt}-\mathrm{N}$ & 254 & -1 & 246 & -9 & 247 & -8 & 255 \\
\hline$\delta \mathrm{N}-\mathrm{Pt}-\mathrm{Cl}$ & 208 & -1 & 209 & 0 & 213 & 4 & 210 \\
\hline$\delta \mathrm{Cl}-\mathrm{Pt}-\mathrm{Cl}$ & 162 & 0 & 164 & 2 & 161 & -1 & 162 \\
\hline $\mathrm{rms}^{h}(\%)$ & 1.1 & & 1.3 & & 1.5 & & \\
\hline CPUT $^{i}$ & $00: 20$ & & $06: 28$ & & $06: 22$ & & \\
\hline
\end{tabular}

${ }^{a} v_{\mathrm{s}}$ and $\nu_{\mathrm{as}}$ stand for symmetric and antisymmetric stretching modes, respectively; $\delta_{\mathrm{s}}$ and $\delta_{\mathrm{as}}$ stand for symmetric and antisymmetric deformation modes, respectively; $\delta=$ skeletal in-plane deformation modes; $\rho$ and $\rho^{\prime}$ stand for in-phase and out-of-phase rocking modes, respectively. For a schematic representation of the $\delta \mathrm{N}-\mathrm{Pt}-\mathrm{N}, \delta \mathrm{Cl}-$ $\mathrm{Pt}-\mathrm{N}$, and $\delta \mathrm{Cl}-\mathrm{Pt}-\mathrm{Cl}$ modes refer to Figure 3 of ref 9. ${ }^{b}$ Theoretical scaled value $\left(\lambda_{1}=0.85\left(\nu \mathrm{NH}_{3}\right.\right.$ and $\left.\delta \mathrm{NH}_{3}\right), \lambda_{2}=0.93\left(\rho \mathrm{NH}_{3}\right.$ and $\delta \mathrm{N}-$ $\mathrm{Pt}-\mathrm{Cl}), \lambda_{3}=1.04\left(v \mathrm{Pt}-\mathrm{N}, v \mathrm{Pt}-\mathrm{Cl}, \delta \mathrm{N}-\mathrm{Pt}-\mathrm{N}\right.$, and $\left.\delta \mathrm{Cl}-\mathrm{Pt}-\mathrm{C}_{1}\right)$ (Table 5). ${ }^{c}$ Deviation from experimental after scaling (theoretical $(\omega)$ - experimental $(v)) .{ }^{d}$ Theoretical scaled value $\left(\lambda_{1}=0.93\left(\nu \mathrm{NH}_{3}\right.\right.$, $\delta_{\mathrm{as}} \mathrm{NH}_{3}$, and $\left.\delta \mathrm{N}-\mathrm{Pt}-\mathrm{Cl}\right), \lambda_{2}=0.98\left(\rho \mathrm{NH}_{3}\right.$ and $\left.\delta_{\mathrm{s}} \mathrm{NH}_{3}\right), \lambda_{3}=1.05$ $\left(v \mathrm{Pt}-\mathrm{N}, v \mathrm{Pt}-\mathrm{Cl}, \delta \mathrm{N}_{1}-\mathrm{Pt}-\mathrm{N}\right.$, and $\left.\delta \mathrm{Cl}-\mathrm{Pt}-\mathrm{Cl}\right)($ Table 5$) .{ }^{e}$ Theoretical scaled value $\left(\lambda_{1}=0.91\left(\nu \mathrm{NH}_{3}, \delta_{\mathrm{as}} \mathrm{NH}_{3}\right.\right.$, and $\left.\delta \mathrm{N}-\mathrm{Pt}-\mathrm{Cl}\right), \lambda_{2}=0.96$ $\left(\rho \mathrm{NH}_{3}\right.$ and $\left.\delta_{\mathrm{s}} \mathrm{NH}_{3}\right), \lambda_{3}=1.01\left(\nu \mathrm{Pt}-\mathrm{N}, \nu \mathrm{Pt}-\mathrm{Cl}, \delta \mathrm{N}_{\mathrm{l}}-\mathrm{Pt}-\mathrm{N}\right.$, and $\delta \mathrm{Cl}-$ $\mathrm{Pt}-\mathrm{Cl}$ ) (Table 5). ${ }^{f}$ Experimental FT-Raman frequencies. ${ }^{9}{ }^{g}$ Observed in the FTIR spectra; probably strongly overlapped with the other $\delta \mathrm{NH}_{3}$ modes in the Raman spectrum. ${ }^{h}$ Calculated with equation: ${ }^{9} \mathrm{rms}=$ $\left(\sum_{i=1}^{n} \Delta_{i}\right) / n .{ }^{i}$ Computational time required for a frequency calculation, min:sec.

both the rms and CPUT criteria, ECP1 (with no polarization functions) appears to be the best choice, independently of the theoretical method used (either HF or DFT).

At this point, it may be concluded that the best prediction of the cisplatin experimental vibrational frequencies is obtained using a three-factor scaling, without polarization functions. Additionally, this approach leads to the lowest computational cost.

Table 6 comprises the calculated scaled vibrational frequencies for cDDP (above $160 \mathrm{~cm}^{-1}$ ), obtained in the light of the best accuracy/CPUT relationship, chosen from Table 5-HFECP1, B3L-ECP1 and mPW1-ECP1-along with the experimental FT-Raman wavenumbers. Careful analysis of this data evidence that the major deviations occur for wavenumbers above $700 \mathrm{~cm}^{-1}$, the $\mathrm{N}-\mathrm{H} \cdots \mathrm{Cl}$ interactions being the most likely reason for these shifts. It should not be forgotten, however, that all calculations are performed for the isolated molecule, while the experimental spectra are obtained in the solid state, where the intra- and intermolecular interactions play an essential role. As for the angles of the molecule, these deviations are probably diminished if the effect of the intermolecular $\mathrm{N}-\mathrm{H} \cdots \mathrm{Cl}$ contacts (by considering dimeric structures) are taken into account.
Interestingly, the HF-ECP1 combination displays the best accuracy in the prediction of the cisplatin vibrational modes, with the lowest computational cost. Within the DFT methods, $\mathrm{mPW} 1 \mathrm{PW}$ and B3LYP present comparable accuracies, B3LYP being slightly better.

The lower vibrational modes are not considered in this analysis since they are strongly dependent on the interactions present at the solid state and affected by the dispersion of the lattice.

\section{Conclusions}

In this work, a conformational and vibrational analysis of the anticancer drug cisplatin was performed. Following a reported study aiming at determining the best theoretical combination for the description of this type of $\mathrm{Pt}(\mathrm{II})$ compounds, with the best accuracy/computational cost ratio, ${ }^{9}$ the ECP approach was presently tested in order to compare with the previous AE results. For the majority of the theoretical approaches undertaken, only one real minimum was found from the three possible geometries for the isolated molecule. This conformer was selected to undergo a thorough structural and vibrational analysis.

HF and the mPW1PW DFT functional were shown to be the best protocols to perform a cDDP structural analysis, while the widely used B3LYP functional led to the worse results. For predicting the experimental vibrational spectra above $160 \mathrm{~cm}^{-1}$, HF showed the best performance, yielding the minimum error at the lowest computational cost. However, this method was not totally consistent for the very low frequencies modes, as opposed to the density functionals, which displayed a clear advantage in this spectral region. Furthermore, although the HF approach has shown to be quite appropriate, with a low computational cost, it has to be kept in mind that if there is no reliability regarding the most stable cDDP structure, the whole study looses its purpose even if it can be achieved at a low computational cost.

On the basis of the data obtained for both the structural and vibrational calculations, it appears that mPW1PW is the best method for an accurate and inexpensive representation of cDDP.

Regarding the ECPs performances, ECP1 (LANL2MB) yielded the best vibrational results and ECP3 (CEP-4G) is responsible for the best structural parameters (Table 7), both representing clear advantages over ECP2 (LANL2DZ), although the latter has been previously reported ${ }^{25}$ to yield good results for the prediction of the structural and vibrational cisplatin parameters.

As to the improvement in the description of the atoms' valence shell, it was shown that the presence of polarization functions is very important for a suitable prediction of the structural parameters, while the best vibrational results were obtained without inclusion of polarization functions. Splitting of the orbitals leads in general, to a worsening of the results.

The combination [theoretical method/ECP/valence shell improvement] will thus have to be chosen in accordance with the type of analysis aimed at.

If only a structural study is aimed, not many problems arise, but in the case of a complete conformational analysis one feature will have to be sacrificed-either the accuracy or the computational cost-and we will have to find a compromise between the two.

Most of the deviations observed in the present paper result from the absence of the effects of the intermolecular $\mathrm{N}-\mathrm{H} \cdots$ $\mathrm{Cl}$ interactions, known to affect significantly both the structural parameters and vibrational frequencies. In order to overcome 
TABLE 7: Compilation of the Best Results (Best Accuracy/ Computational Cost Relation) Obtained for the Structural Parameters for the Lowest Energy Minimum Predicted for cDDP (Geometry cDDP1) Using the ECPs Approximation and the All-Electrons (AE) Calculations for Comparison

\begin{tabular}{|c|c|c|c|c|c|c|}
\hline \multicolumn{7}{|c|}{ Structural Parameters } \\
\hline $\begin{array}{c}\text { theoretical } \\
\text { level }\end{array}$ & $\begin{array}{l}\text { polarization } \\
\text { function }^{a}\end{array}$ & $\Delta \Delta^{b}$ & $\mathrm{CPUT}^{d}$ & $\begin{array}{l}\text { theoretical } \\
\text { level }^{e}\end{array}$ & $\Delta \Delta^{e}$ & $\mathrm{CPU}$ \\
\hline HF-ECP1 & yes/yes & 5.5 & $02: 10$ & HF-AE4(f) & 5.0 & $07:($ \\
\hline HF-ECP3 & yes/yes & 5.1 & $02: 12$ & & & \\
\hline B3L-ECP1 & yes/yes & 6.2 & $12: 44$ & B3L-AE4(f) & 5.6 & $23: 0$ \\
\hline B3L-ECP3 & yes/yes & 5.6 & $12: 53$ & & & \\
\hline mPW1-ECP1 & yes/yes & 5.8 & $12: 54$ & mPW1-AE4(f) & 5.2 & $24: 0$ \\
\hline mPW1-ECP3 & yes/yes & 5.2 & $13: 01$ & & & \\
\hline
\end{tabular}

\section{Vibrational Analysis}

\begin{tabular}{lllllll}
\hline $\begin{array}{c}\text { theoretical } \\
\text { level }\end{array}$ & $\begin{array}{c}\text { polarization } \\
\text { function }^{a}\end{array}$ & $\begin{array}{c}\mathrm{rms}^{c} \\
(\%)\end{array}$ & CPUT $^{d}$ & $\begin{array}{c}\text { theoretical } \\
\text { level }^{e}\end{array}$ & $\begin{array}{c}\mathrm{rms}^{e} \\
(\%)\end{array}$ & CPUT $^{e}$ \\
\hline HF-ECP1 & no/no & 1.1 & $00: 20$ & HF-AE4(f) & 1.5 & $07: 08$ \\
HF-ECP3 & no/yes & 1.6 & $00: 36$ & & & \\
B3L-ECP1 & no/no & 1.3 & $06: 28$ & B3L-AE4(f) & 1.3 & $23: 07$ \\
B3L-ECP3 & no/yes & 1.8 & $08: 24$ & & & \\
mPW1-ECP1 & no/no & 1.5 & $06: 22$ & mPW1-AE1 & 1.7 & $15: 00$ \\
mPW1-ECP3 & no/yes & 1.6 & $08: 30$ & & &
\end{tabular}

${ }^{a}$ no/no (no polarization functions), no/yes (polarization function at the $\mathrm{Pt}$ atom) yes/yes (polarization functions at all heavy atoms)-using the FineGrid option for the DFT calculations. ${ }^{b}$ Overall deviation values selected from Table 3 (yes/yes). ${ }^{c}$ Values selected from Table S51 (no/ no) and Table S5k (no/yes). ${ }^{d}$ Computational time required for a frequency calculation in min:sec selected from Table 4. ${ }^{e}$ Selected theoretical levels collected from ref 9; (f) indicates the presence of an f-polarization function at the Pt atom (AE1-6-31G(d); AE4-6-311G(d)).

these deficiencies, the dimeric structures of some small Pt(II) molecules held by that type of intermolecular contacts (and evidenced by the X-ray structures of the literature) are under study, namely for cDDP and $\mathrm{Pt}(\mathrm{en}) \mathrm{Cl}_{2}$. However, it has to be kept in mind that as the systems increases to polynuclear systems, for instance, the dimer simulations will quickly become prohibitive. Therefore, the study of the parent isolated molecule is of paramount importance, namely for the obtaining the vibrational scaling factors most appropriate for this type of systems.

Table 7 gathers the results on the best performances of the ECPs and AE studies carried out for cisplatin. It is easily verified that the use of ECPs represents a clear advantage over the AE calculations: considerable reduction of the computational effort (particularly for the DFT protocols), while the accuracy is not largely affected. The most obvious case is the mPW1PW level, which yields even better results when combined to an ECP approach than when coupled to the more demanding AE basis sets.

Acknowledgment. The authors acknowledge financial support from the Portuguese Foundation for Science and Technology-Unidade de Química-Física Molecular and Research Project POCTI/47256/QUI/2002 (co-financed by the european community fund FEDER). S.M.F. also acknowledges a Ph.D. fellowship (SFRH/BD/17493/2004).
Supporting Information Available: Supplementary Tables S2-S51 regarding other results obtained for energies and structural parameters and different scaling schemes for the calculated harmonic frequencies. This material is available free of charge via the Internet at http://pubs.acs.org.

\section{References and Notes} 698.

(1) Rosenberg, B.; Vancamp, L.; Krigas, T. Nature 1965, 205

(2) Calvert, A. H.; Harland, S. J.; Newell, D. R.; Siddik, Z. H.; Jones, A. C.; McElwain, T. J.; Raju, S.; Wiltshaw, E.; Smith, I. E.; Baker, J. M.; Peckham, M. J.; Harrap, K. R. Cancer. Chemother. Pharmacol. 1982, 9, 140.

(3) Kidani, Y.; Noji, M.; Tashiro, T. Gann. 1980, 71, 637.

(4) Manzotti, C.; Pratesi, G.; Menta, E.; Di Domenico, R.; Cavalletti, E.; Fiebig, H. H.; Kelland, L. R.; Farrell, N.; Polizzi, D.; Supino, R.; Pezzoni, G.; Zunino, F. Clin. Cancer Res. 2000, 6, 2626.

(5) Galanski, M.; Arion, V. B.; Jakupec, M. A.; Keppler, B. K. Curr. Pharm. Des. 2003, 9, 2078

(6) Schmitt, M.; Popp, J. J. Raman Spectrosc. 2006, 37, 20.

(7) Nolasco, M. M.; Amado, A. M.; Ribeiro-Claro, P. J. A. Chem. Phys. Chem 2006, 7, 2150.

(8) Amado, A. M.; Otero, J. C.; Marques, M. P. M.; Batista de Carvalho, L. A. E. Chem. Phys. Chem 2004, 5, 1.

(9) Amado, A. M.; Fiúza, S. M.; Marques, M. P. M.; Batista de Carvalho, L. A. E. J. Chem. Phys. 2007, 127, 185104.

(10) Dolg, M. Effective Core Potentials. In Modern Methods and Algorithms of Quantum Chemistry, 2nd ed.; Grotendorst, J., Ed.; John von Neumann Institute for Computing: Julich, Germany, 2000; Vol. 3.

(11) Frisch, M. J.; Trucks, G. W.; Schlegel, H. B.; Scuseria, G. E.; Robb, M. A.; Cheeseman, J. R.; Montgomery, J. A., Jr.; Vreven, T.; Kudin, K. N.; Burant, J. C.; Millam, J. M.; Iyengar, S. S.; Tomasi, J.; Barone, V.; Mennucci, B.; Cossi, M.; Scalmani, G.; Rega, N.; Petersson, G. A.; Nakatsuji, H.; Hada, M.; Ehara, M.; Toyota, K.; Fukuda, R.; Hasegawa, J.; Ishida, M.; Nakajima, T.; Honda, Y.; Kitao, O.; Nakai, H.; Klene, M.; Li, X.; Knox, J. E.; Hratchian, H. P.; Cross, J. B.; Bakken, V.; Adamo, C.; Jaramillo, J.; Gomperts, R.; Stratmann, R. E.; Yazyev, O.; Austin, A. J ; Cammi, R.; Pomelli, C.; Ochterski, J. W.; Ayala, P. Y.; Morokuma, K.; Voth, G. A.; Salvador, P.; Dannenberg, J. J.; Zakrzewski, V. G.; Dapprich, S.; Daniels, A. D.; Strain, M. C.; Farkas, O.; Malick, D. K.; Rabuck, A. D.; Raghavachari, K.; Foresman, J. B.; Ortiz, J. V.; Cui, Q.; Baboul, A. G.; Clifford, S.; Cioslowski, J.; Stefanov, B. B.; Liu, G.; Liashenko, A.; Piskorz, P.; Komaromi, I.; Martin, R. L.; Fox, D. J.; Keith, T.; Al-Laham, M. A.; Peng, C. Y.; Nanayakkara, A.; Challacombe, M.; Gill, P. M. W.; Johnson, B.; Chen, W.; Wong, M. W.; Gonzalez, C.; Pople, J. A. Gaussian 03, Revision D.01; Gaussian, Inc.: Wallingford CT, 2004. 2657.

(12) Hehre, W. J.; Stewart, R. F.; Pople, J. A. J. Chem. Phys. 1969, 51,

(13) Hay, P. J.; Wadt, W. R. J. Chem. Phys. 1985, 82, 284.

(14) Stevens, W.; Basch, H.; Krauss, J. J. Chem. Phys. 1984, 81, 6026.

(15) Dunning, T. H., Jr.; Hay, P. J. Modern Theoretical Chemistry; Schaefer, H. F., III, Ed.; Plenum: New York, 1976; Vol. 3.

(16) Hay, P. J.; Wadt, W. R. J. Chem. Phys. 1985, 82, 299.

(17) Amado, A. M.; Ribeiro-Claro, P. J. A. J. Inorg. Biochem. 2004, $98,561$.

(18) Hehre, W. J.; Radom, L.; Schleyer, P. v. R.; Pople, J. A. Ab Initio Molecular Orbital Theory; Wiley-Interscience Publication: New York, 1986.

(19) Chermette, H. Coord. Chem. Rev. 1998, 699, 178-180.

(20) Becke, A. D. Phys. Rev. A 1988, 38, 3098.

(21) Lee, C. T.; Yang, W. T.; Parr, R. G. Phys. Rev. B 1988, 37 , 785.

(22) Adamo, C.; Barone, V. J. Chem. Phys. 1998, 108, 664

(23) Perdew, J. P.; Burke, K.; Ernzerhof, M. Phys. Rev. Lett. 1996, 77, 3865 .

(24) Scott, A. P.; Radom, L. J. Phys. Chem. 1996, 100, 16502.

(25) Wysokinsky, R.; Michalska, D. J. Comp. Chem. 2001, 22 901.

(26) Milburn, G. H. W.; Truter, M. R.; J. Chem. Soc. 1966, 11, 1609 